ARTIGO ORIGINAL ORIGINAL ARTICLE

\title{
Qualidade metodológica das avaliações econômicas de antineoplásicos submetidas à Agência Nacional de Saúde Suplementar (ANS) na atualização do rol 2020 - Estudo de avaliação crítica
}

\author{
Methodological quality of economic assessments of \\ antineoplastics submitted to Supplementary Health Agency \\ in the update of the 2020 list - Critical assessment study
}

Ângela Maria Bagattini 1,2,3, Daniela Vianna Pachito ${ }^{1,4}$,

Rafael Leite Pacheco ${ }^{1,5,6,7}$, Aline Rocha ${ }^{7,8}$, Rachel Riera ${ }^{1,5,6,7}$

DOI: 10.21115/JBES.v13.n1.p31-42

\section{Palavras-chave:}

economia da saúde, avaliação de custo-efetividade, antineoplásicos, neoplasias, tomada de decisões, avaliação crítica da literatura

\section{Keywords}

health economics, cost-effectiveness evaluation, antineoplastic agents, neoplasms, decision making, critical appraisal

\section{RESUMO}

Objetivo: Descrever e analisar criticamente as avaliações econômicas de medicamentos antineoplásicos submetidas à Agência Nacional de Saúde Suplementar (ANS) durante o processo de atualização do rol de procedimentos em saúde 2020. Métodos: Estudo transversal de análise crítica dos estudos de avaliação econômica integrantes da documentação submetida à ANS com o objetivo de incorporação no rol de procedimentos. A avaliação da qualidade metodológica foi realizada por meio da ferramenta Methodology Checklist 6: Economic Evaluations Version 3.0 da Scottish Intercollegiate Guidelines Network. Resultados: Foram incluídas 49 avaliações econômicas: 22 estudos de custo-efetividade, 10 estudos de custo-utilidade, três estudos de custo-minimização e 14 estudos de custo-efetividade e custo-utilidade. A qualidade metodológica foi considerada, na maior parte (88\%), como aceitável ou de baixa qualidade. Conclusão: Estudos de avaliação econômica são fundamentais no processo decisório de incorporação de tecnologias na saúde suplementar. Esta análise crítica sugere que a qualidade dos estudos econômicos apresentados dentro das propostas de incorporação de antineoplásicos durante o processo de atualização do rol 2020 da ANS foi limitada. Inconsistências metodológicas e falta de um relato transparente reduzem a validade e a aplicabilidade dos achados na tomada de decisão.

\section{ABSTRACT}

Objective: To describe and critically appraise the economic evaluations of antineoplastic drugs submitted to the ANS during the process of updating its 2020' list of procedures. Methods: Cross-sectional study of critical analysis of the economic evaluation studies included in the documentation submitted to the ANS with the aim of incorporating them into the list of procedures. The methodological quality assessment was carried out using the Methodology Checklist 6: Economic Evaluations Version 3.0 of the Scottish Intercollegiate Guidelines Network. Results:

\footnotetext{
Recebido em: 17/01/2021. Aprovado para publicação em: 25/02/2021.

1. Núcleo de Avaliação de Tecnologias em Saúde, Hospital Sírio-Libanês (NATS-HSL), São Paulo, SP, Brasil.

2. Universidade Federal de Goiás, Instituto de Patologia Tropical e Saúde Pública, Goiânia, GO, Brasil.

3. Instituto Nacional de Ciência e Tecnologia de Avaliação em Tecnologias em Saúde (INCT/IATS), Porto Alegre, RS, Brasil.

4. Fundação Getúlio Vargas (FGV), São Paulo, SP, Brasil.

5. Centro Universitário São Camilo (CUSC), São Paulo, SP, Brasil.

6. Oxford-Brasil EBM Alliance.

7. Escola Paulista de Medicina, Universidade Federal de São Paulo (Unifesp), São Paulo, SP, Brasil.

8. Cochrane Brasil, São Paulo, SP, Brasil.

Financiamento: Este estudo foi apoiado pelo Ministério da Saúde do Brasil pelo Programa de Apoio ao Desenvolvimento Institucional do Sistema Único de Saúde (Proadi-SUS), liderado pelo Hospital Sírio-Libanês, São Paulo, SP, Brasil.

Conflito de interesses: Todos os autores declaram não haver interesses financeiros concorrentes relacionados a este manuscrito. Autor correspondente: Rafael Leite Pacheco. Hospital Sírio-Libanês (HSL). Barata Ribeiro, 142, Bela Vista, São Paulo, SP, CEP: 01308-000, Brasil. E-mail: rleitepacheco@hotmail.com.
} 
Overall, 49 economic evaluations were included: 22 cost-effectiveness studies, 10 cost-utility studies, three cost-minimization studies and 14 mixed economic studies. Methodological quality was mostly considered as acceptable or low quality. Conclusion: Economic evaluation studies are fundamental in the decision-making process of incorporating technologies into supplementary health care. This critical appraisal suggests that the quality of the economic studies presented within the proposals to incorporate antineoplastics during the process of updating the ANS 2020 roll was limited. Methodological inconsistencies and lack of transparent reporting reduce the validity and applicability of findings for decision-making.

\section{Introdução}

No Brasil, dois sistemas de saúde coexistem: o sistema público (Sistema Único de Saúde - SUS), com cobertura universal e financiamento público, que atende em torno de $75 \%$ da população, e o sistema de saúde suplementar, regulamentado pela Agência Nacional de Saúde Suplementar (ANS), criada a partir da Lei no 9.656, de 3 de junho de 1998.

O sistema de saúde suplementar conta com 979 operadoras com registro ativo em 2020. Conjuntamente, essas operadoras fornecem assistência à saúde para aproximadamente 47 milhões de pessoas, representando $24,2 \%$ da população brasileira. A proporção populacional coberta pelo sistema de saúde suplementar varia entre os estados na faixa de 5,6\% a $34,8 \%$, com as maiores proporções de cobertura observadas nas regiões Sul e Sudeste (Brasil, s.d.).

A ANS é responsável pela atualização periódica do rol de medicamentos, dispositivos e procedimentos que devem ser minimamente oferecidos pelos planos de saúde aos seus usuários. O processo de atualização do rol foi aprimorado em 2020, adotando métodos consolidados na área de avaliação de tecnologias em saúde (Brasil, 2018).

As propostas de incorporação são recebidas por um sistema on-line, e parte delas se refere a medicamentos antineoplásicos. É reconhecido que as neoplasias contribuem substancialmente com a carga econômica e epidemiológica nos sistemas de saúde no mundo. Do ponto de vista epidemiológico, estima-se que, no mundo, em 2018 tenham ocorrido 18,1 milhões de novos casos e até 9,5 milhões de óbitos por câncer (Bray et al., 2018). No Brasil, 75\% dos óbitos são relacionados a doenças não transmissíveis, entre elas as neoplasias, responsáveis por 130.202 óbitos no Brasil em 2017 (Malta et al., 2020).

O alto custo relacionado aos tratamentos oncológicos impõe um grande desafio para governos e agências de saúde em um contexto de recursos limitados: a incorporação de tecnologias efetivas e seguras, mas que também sejam custo-efetivas e se encaixem no orçamento disponível (Aguiar et al., 2019; Pereira et al., 2019).

O objetivo deste estudo foi descrever e analisar criticamente as avaliações econômicas de medicamentos antineoplásicos submetidas à ANS durante o processo de atualização do rol de procedimentos em saúde 2020.

\section{Métodos}

\section{Desenho e local}

Este foi um estudo transversal de análise crítica de estudos de avaliação econômica de medicamentos antineoplásicos apresentados para embasar pedidos de incorporações no rol de procedimentos da ANS. O estudo foi desenvolvido pelo Núcleo de Avaliação de Tecnologias em Saúde do Hospital Sírio-Libanês.

\section{Busca por avaliações econômicas}

As propostas de incorporação no rol 2020 foram encaminhadas por associação de pacientes, fabricantes das tecnologias avaliadas ou associações médicas, sendo compostas por: (i) avaliação de eficácia e segurança, (ii) avaliação econômica com análise de custo-efetividade ou custo-minimização e (iii) análise de impacto orçamentário.

Os documentos encaminhados pelos proponentes e avaliados pela ANS ficaram disponíveis, em domínio público, no período de 8 de outubro a 21 de novembro de 2020, por meio da Consulta Pública (CP) no 81, no endereço eletrônico da ANS, onde foram analisados os relatórios de medicamentos oncológicos, especificamente as avaliações de custo-efetividade ou custo-minimização realizadas pelos proponentes.

\section{Seleção das avaliações econômicas}

Foram consideradas todas as avaliações econômicas de medicamentos antineoplásicos submetidas à ANS com o objetivo de incorporação no rol de procedimentos. Na situação em que múltiplos proponentes apresentaram a mesma avaliação econômica, apenas uma avaliação foi considerada.

\section{Extração de dados}

Após a leitura do documento completo de cada estudo de avaliação econômica incluído, foram extraídas as seguintes informações: tecnologia avaliada, população a que se destinava a tecnologia, tipos de medidas de efetividade, tipo de avaliação econômica realizada, perspectiva da análise econômica, fontes dos dados, tipo de modelagem, horizonte temporal, dados sobre análises de sensibilidade, taxa de desconto e métodos de custeio.

\section{Avaliação da qualidade metodológica}

A qualidade metodológica dos estudos de avaliação econômica incluídos foi analisada por um único pesquisador, considerando os itens da ferramenta Methodology Checklist 
6: Economic Evaluations Version 3.0 da Scottish Intercollegiate Guidelines Network (SIGN). A ferramenta SIGN é composta por duas partes: (i) nove questões sobre a validade interna e externa, com opção de respostas "Sim", "Não" ou "Não é possível afirmar", e (ii) avaliação geral do estudo, com opção de classificação em "Baixa qualidade", "Aceitável" ou "Alta qualidade" (Harbour, 2012).

\section{Resultados}

Na atualização do rol de procedimentos de cobertura obrigatória pelos planos de saúde 2019-2020, passaram pela avaliação técnica 185 unidade de análise técnica (propostas), sendo 85 de medicamentos e 41 de medicamentos relacionados ao tratamento oncológico.

Algumas tecnologias foram abordadas por mais de uma proposta. Ao final, 49 estudos de avaliação econômica foram incluídos nesta análise crítica: 22 estudos de custo-efetividade, 10 estudos de custo-utilidade, três estudos de custo-minimização e 14 estudos apresentaram simultaneamente análise de custo-efetividade e custo-utilidade, dessa forma, realizaram simultaneamente análises com desfechos de efetividade e utilidade e apresentaram diferentes razões de custo-efetividade incremental (RCEls) para cada medida de efetividade utilizada (Figura 1).

Todas os estudos de e custo-efetividade ou custo-utilidade adotaram métodos de modelagem, e o modelo de sobrevida particionado foi o método mais utilizado (27/49, 59\%). Três estudos não descreveram o tipo de modelagem utilizado (Figura 2).

Todas as avaliações econômicas foram realizadas sob a perspectiva do sistema suplementar de saúde e utilizaram apenas custos diretos. A taxa de desconto utilizada em maioria (44) foi de 5\%, e apenas as avaliações que utilizaram um horizonte temporal curto (5) não incluíram taxas de desconto.

A maior parte dos estudos utilizou a expectativa de vida como horizonte temporal e 10 estudos assumiram um horizonte temporal de 20 anos. O horizonte temporal variou de um mês a 40 anos (Tabela 1).

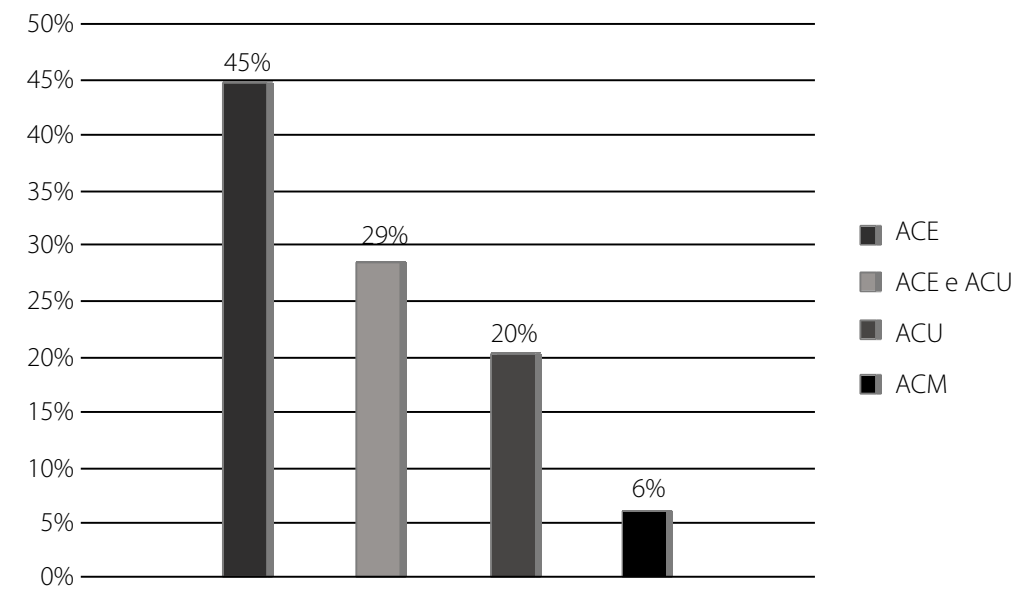

ACE: análise de custo-efetividade; ACU: análise de custo-utilidade; ACM: análise de custo-minimização.

Figura 1. Tipo de avaliação econômica apresentada nas propostas de incorporação de medicamentos antineoplásicos - Rol ANS 2020

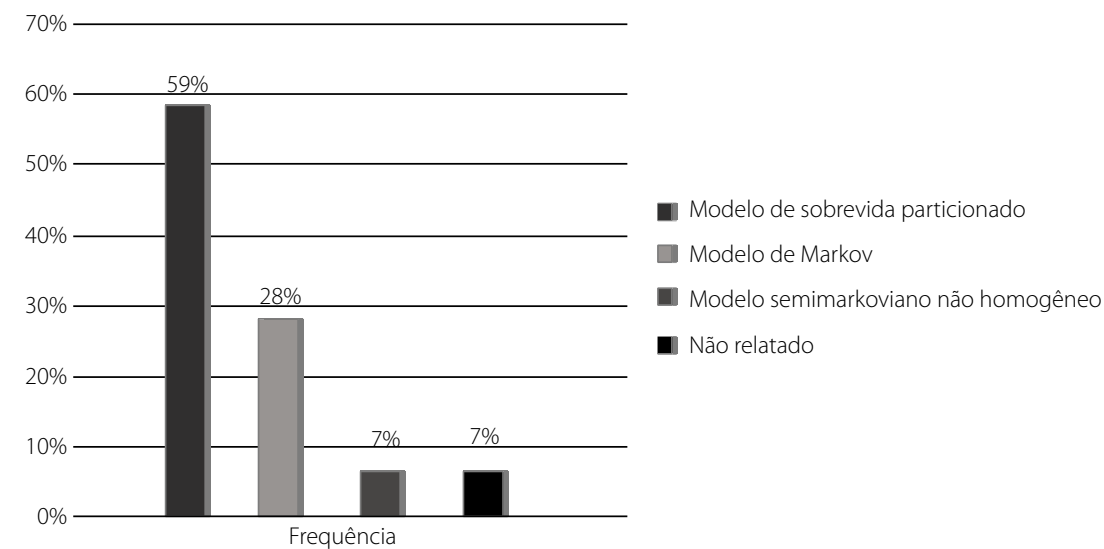

Figura 2. Tipo de modelagem utilizada nas análises econômicas apresentadas nas propostas de incorporação de medicamentos antineoplásicos - Rol ANS 2020 


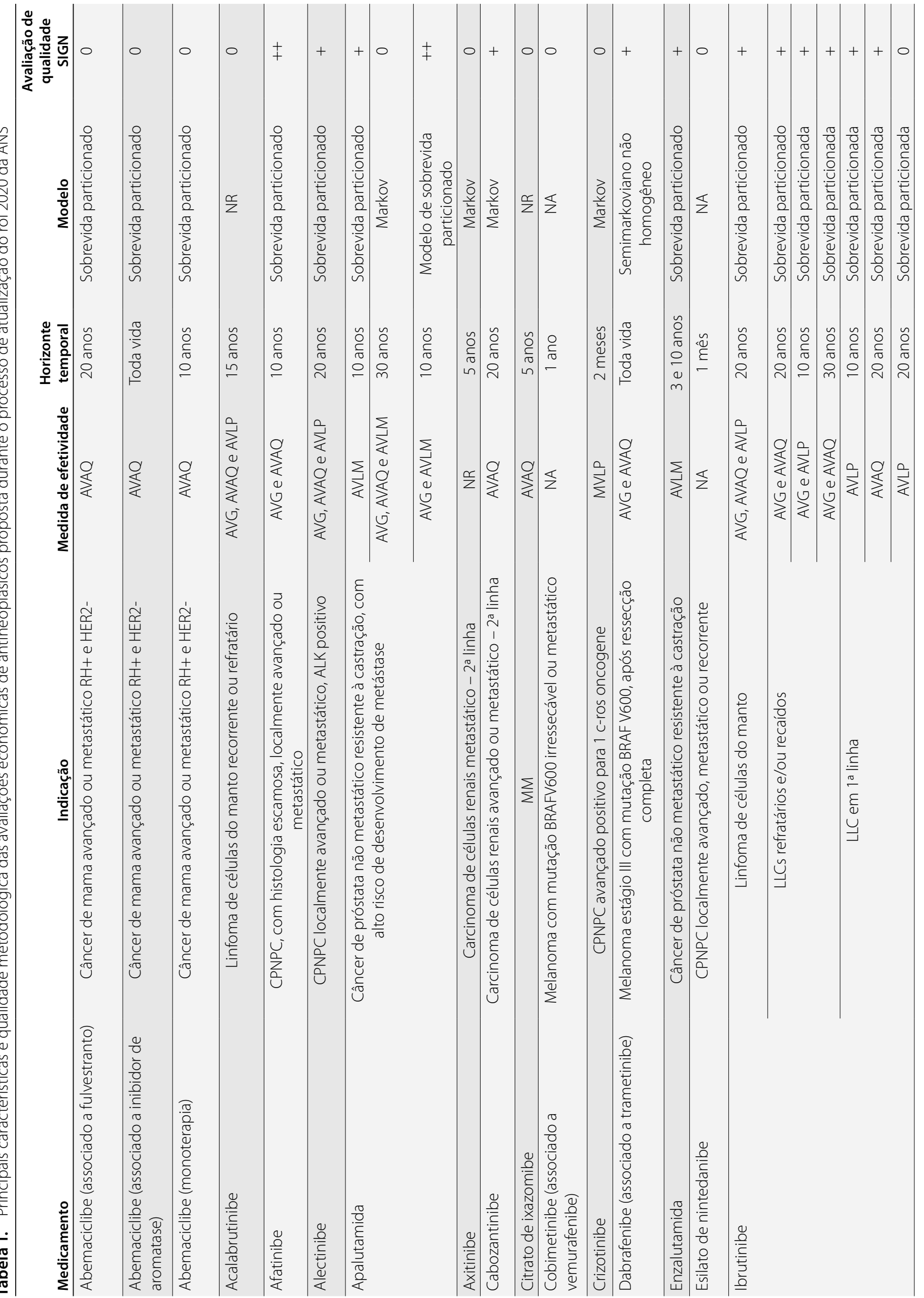




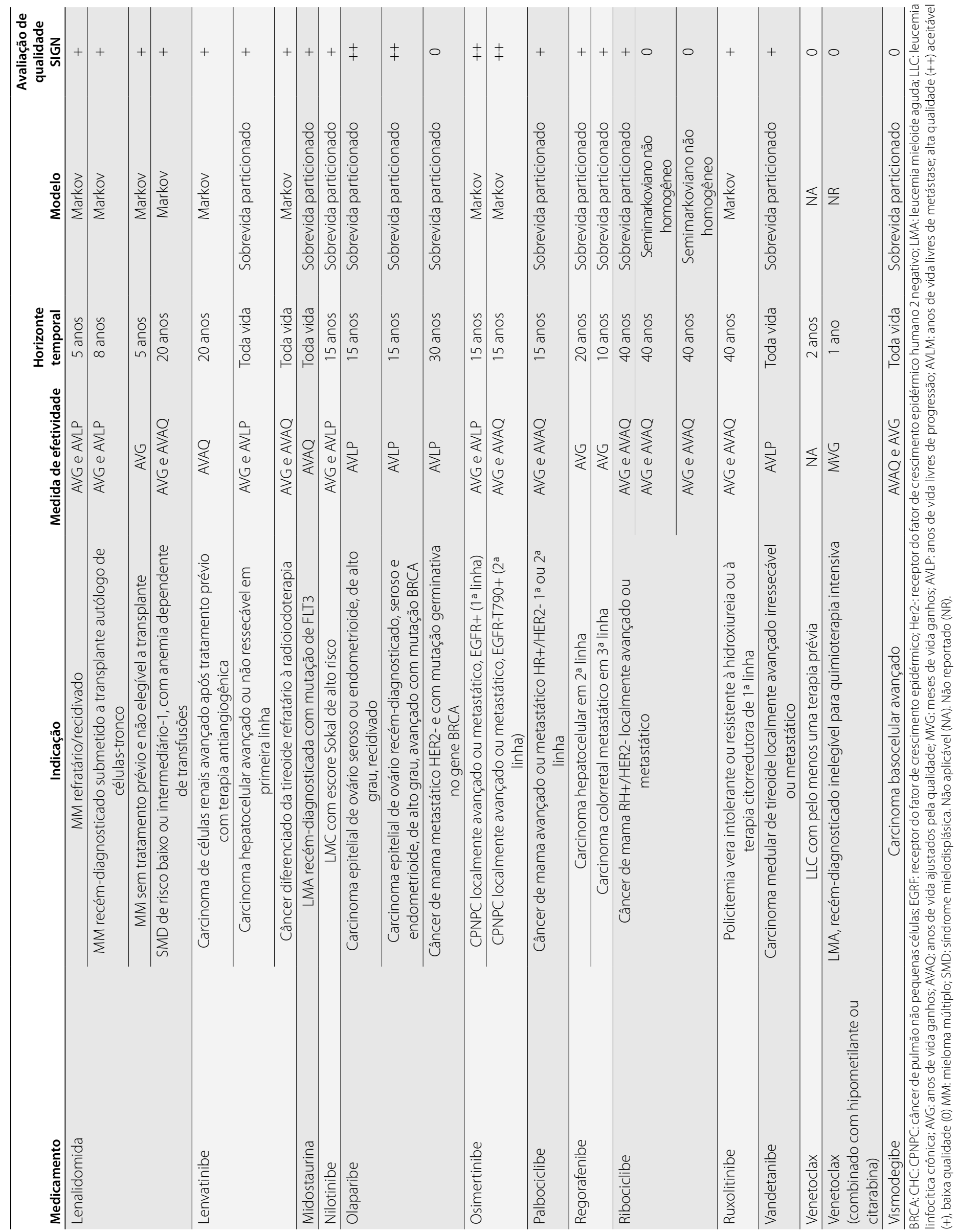


Análises de sensibilidade foram realizadas em 27 (55\%) estudos: 10 realizaram análises probabilísticas e determinísticas, 9 (18\%), apenas determinística e 8 (16\%), apenas probabilística; cinco propostas não relataram ou apresentaram as análises de sensibilidade.

As seguintes medidas de efetividade foram utilizadas pelos estudos incluídos: anos de vida ganhos (45\%), anos de vida ajustados pela qualidade (45\%), anos de vida livres de progressão (28\%), anos de vida livres de metástase (4\%), meses de vida livres de progressão (2\%) e meses de vida ganhos (2\%).

Os estudos econômicos avaliaram mais frequentemente os seguintes antineoplásicos: lenalidomida $(n=4)$, ibrutinibe $(n=3)$, abemaciclibe $(n=3)$, lenvatinibe $(n=3)$ e olaparibe $(n=3)$. As doenças oncológicas mais frequentemente avaliadas pelos estudos foram: câncer de mama $(n=6)$, câncer de pulmão não pequenas células $(n=6)$, e mieloma múltiplo ( $n$ =4), das quais três eram relacionadas à lenalidomida.

A qualidade metodológica (Material suplementar) dos estudos de avaliação econômica (Harbour, 2012) foi considerada alta para seis estudos, aceitável para 27 estudos (significando que o estudo contém algumas falhas metodológicas associadas a risco de viés) e baixa para 16 estudos (significando que a maioria dos critérios não foi atendida ou que há falhas significativas relacionadas aos principais aspectos do desenho do estudo). Esses resultados demonstram que a maioria dos critérios não foi atendida ou que existem falhas metodológicas significativas relacionadas aos principais aspectos do desenho do estudo. Os maiores problemas encontrados foram relacionados à falta de descrição detalhada das fontes de custos utilizadas, seguida da falta de transparência em relação às fontes utilizadas para a estimativa e justificativa dos desfechos utilizados na análise. Grande parte dos estudos não apresentou detalhadamente a pergunta e a justificativa do estudo e o modelo utilizado.

\section{Discussão}

Este estudo descreve e analisa criticamente a qualidade dos estudos de avaliação econômica de medicamentos antineoplásicos propostos à ANS como parte do processo de atualização do rol 2020.

O tipo de avaliação econômica mais frequentemente utilizada foi a análise de custo-utilidade. Esse fato está em alinhamento com as recomendações de agências de saúde e painéis de especialistas (Weinstein et al., 1996), que sugerem, como medida de efetividade, instrumentos que levam em consideração a perspectiva do paciente, o impacto da tecnologia não apenas no tempo de vida, mas também na qualidade de vida relacionada à saúde (AVAQ). Em oncologia, a avaliação do impacto das tecnologias em saúde na qualidade de vida relacionada à saúde é especialmente importante para as doenças avançadas que estão sob cuidados paliativos (Ribeiro et al., 2020)
A utilização de medidas de utilidade impõe maior dificuldade pela escassez de dados locais adequados (Brazier et al., 2019). As avaliações econômicas submetidas à ANS não utilizaram dados nacionais de utilidade, representando uma limitação para a sua validade externa principalmente no contexto de países de renda média como o Brasil. A utilização de dados de outra população pode ser inadequada, devido a fatores culturais e questões econômicas peculiares a cada país (Drummond et al., 2015).

Entre as avaliações econômicas analisadas, foram identificados três estudos de custo-minimização, que são considerados métodos apropriados apenas quando existem evidências robustas de que as tecnologias comparadas possuem a mesma efetividade, premissa essa não atendida nas avaliações apresentadas.

As análises de custo-efetividade utilizaram uma variedade de abordagens de modelagem, como árvores de decisão, modelos de Markov e modelos semimarkovianos, tendo cada modelo suas vantagens e desvantagens. Em nosso recorte para antineoplásicos, a maior parte das avaliações de custo-efetividade utilizou modelo de sobrevida particionado, o que difere de estudo anterior que analisou avaliações econômicas de agentes antineoplásicos apresentados à Comissão Nacional de Incorporação de Tecnologias no SUS (Conitec) (Ribeiro et al., 2020). Por outro lado, nossos achados demonstram alinhamento com as metodologias utilizadas para as avaliações de tecnologias oncológicas, realizadas pela agência de saúde da Inglaterra, com os estudos desenvolvidos pelo National Institute for Health and Care Excellence (NICE), que vem adotando progressivamente esse tipo de modelo para neoplasias avançadas ou metastáticas (Woods et al., 2017).

Em relação à qualidade metodológica, 88\% dos estudos de avaliação econômica foram classificadas como tendo qualidade aceitável ou baixa, apesar da recomendação de que os estudos seguissem as Diretrizes Metodológicas: Diretriz de Avaliações Econômicas do Ministério da Saúde (Brasil, 2014). A maioria dos documentos não apresentou a justificativa para a elaboração da análise e a escolha do modelo, e em muitos casos não foi possível identificar claramente a fonte dos dados de custos e de efetividade. Assim como já relatado em estudo anterior (Ribeiro et al., 2020), alguns estudos não relataram de modo apropriado o modelo econômico e as análises de sensibilidade utilizadas. Entre as limitações encontradas, destaca-se a impossibilidade de acesso às planilhas com os modelos econômicos, o que impediu avaliações mais aprofundadas da estrutura e parametrização dos modelos apresentados. Adicionalmente, destaca-se que a avaliação de qualidade metodológica dos estudos foi realizada por um único avaliador, o que pode ter conferido alguma imprecisão à avaliação.

O crescente avanço tecnológico, principalmente na área de oncologia, exige definições de critérios de priorização na incorporação de novas tecnologias. Para a regulamentação 
dos planos de saúde do sistema de saúde suplementar, papel executado pela ANS, faz-se necessário encontrar um equilíbrio que garanta que as operadoras de saúde disponibilizem tecnologias com efetividade comprovada aos seus usuários, mas que os custos dispendidos não ameacem a viabilidade econômica das operadoras de saúde. As avaliações econômicas têm se tornado cada vez mais importantes para auxiliar os tomadores de decisão na gestão dos custos dessas tecnologias, seja nos sistemas de saúde pública, seja nos sistemas de saúde suplementar, mas ainda são necessárias muitas meIhorias, visto que a maior parte das avaliações econômicas apresentadas para incorporação apresentam falhas na descrição dos dados de forma transparente e falhas metodológicas, prejudicando a sua validade na tomada de decisão.

\section{Conclusões}

Estudos de avaliação econômica são fundamentais no processo decisório de incorporação de tecnologias na saúde suplementar. Esta análise crítica sugere que a qualidade dos 49 estudos econômicos apresentados sobre as propostas de incorporação de antineoplásicos durante o processo de atualização do rol 2020 da ANS foi limitada. Inconsistências metodológicas e falta de um relato transparente reduzem a validade e a aplicabilidade dos achados na tomada de decisão.

\section{Agradecimentos}

Os autores agradecem à equipe de especialistas da ANS envolvidos na atualização do rol 2020.

\section{Referências bibliográficas}

Aguiar PN Jr, Adashek JJ, Roitberg F, Noia Barreto CM, Del Giglio A, Lopes GL Jr. In the Era of Cost-Effectiveness Analysis, Affordability Is a Limiting Factor for Patients' Access to Innovative Cancer Treatments. Value Health Reg Issues. 2019;20:47-50.

Brasil. Agência Nacional de Saúde Suplementar - ANS. Resolução Normativa - RN no 439, de 3 de dezembro de 2018. Dispõe sobre processo de atualização periódica do Rol de Procedimentos e Eventos em Saúde, no âmbito da Agência Nacional de Saúde Suplementar. Disponível em: http://www.ans.gov.br/component/legislacao/?view=legislacao\&task=TextoLei\&format=raw\&id=MzY1Nw==. Acesso em: 30 dez. 2020.

Brasil. Dados e indicadores - Sistema de Informações de Beneficiários - SIB/ ANS/MS e População - IBGE/Datasus. s.d. Disponível em: http://www. ans.gov.br/anstabnet/notas_taxa_cobertura.htm. Acesso em: 5 dez. 2020.

Brasil. Diretrizes Metodológicas: Diretrizes de Avaliação econômica. 2a ed. Brasília: Ministério da Saúde: Ministério da Saúde; 2014.

Bray F, Ferlay J, Soerjomataram I, Siegel RL, Torre LA, Jemal A. Global cancer statistics 2018: GLOBOCAN estimates of incidence and mortality worldwide for 36 cancers in 185 countries. CA Cancer J Clin. 2018;68(6):394-424.

Brazier J, Ara R, Azzabi I, Busschbach J, Chevrou-Séverac H, Crawford B, et al. Identification, Review, and Use of Health State Utilities in CostEffectiveness Models: An ISPOR Good Practices for Outcomes Research Task Force Report. Value Health. 2019;22(3):267-275.

Drummond M, Augustovski F, Kaló Z, Yang BM, Pichon-Riviere A, Bae EY, et al. Challenges faced in transferring economic evaluations to middle income countries. Int J Technol Assess Health Care. 2015;31(6):442-8.

Harbour R. SIGN: Methodology Checklist 6-Economic Evaluations. 2012. Disponivel em: https://www.sign.ac.uk/what-we-do/methodology/ checklists/. Acesso em: 5 out. 2020.

Malta DC, Duncan BB, Schmidt MI, Teixeira R, Ribeiro ALP, Felisbino-Mendes $M S$, et al. Trends in mortality due to non-communicable diseases in the Brazilian adult population: national and subnational estimates and projections for 2030. Popul Health Metr. 2020;18(Suppl 1):16.

Pereira LC, Sturzenegger DVR, Ortiz J, Ayad NME, Cortopassi WA, Safatle LP, et al. Challenges in the Regulation of High-Cost Treatments: An Overview From Brazil. Value Health Reg Issues. 2019;20:191-5.

Ribeiro T, Nobre RM, Campino AC. Avaliação crítica de estudos de custoefetividade de medicamentos oncológicos recomendados para incorporação pela Conitec no Brasil. J Bras Econ Saúde. 2020;12(2):155-63.

Weinstein MC, Siegel JE, Gold MR, Kamlet MS, Russell LB. Recommendations of the Panel on Cost-effectiveness in Health and Medicine. JAMA. 1996;276(15):1253-8.

Woods B, Sideris E, Palmer S, Latimer N, Soares M. NICE DSU Technical Support Document 19. Partitioned Survival Analysis for Decision Modelling in Health Care: A Critical Review. 2017. Disponível em: http:// www.nicedsu.org.uk. 


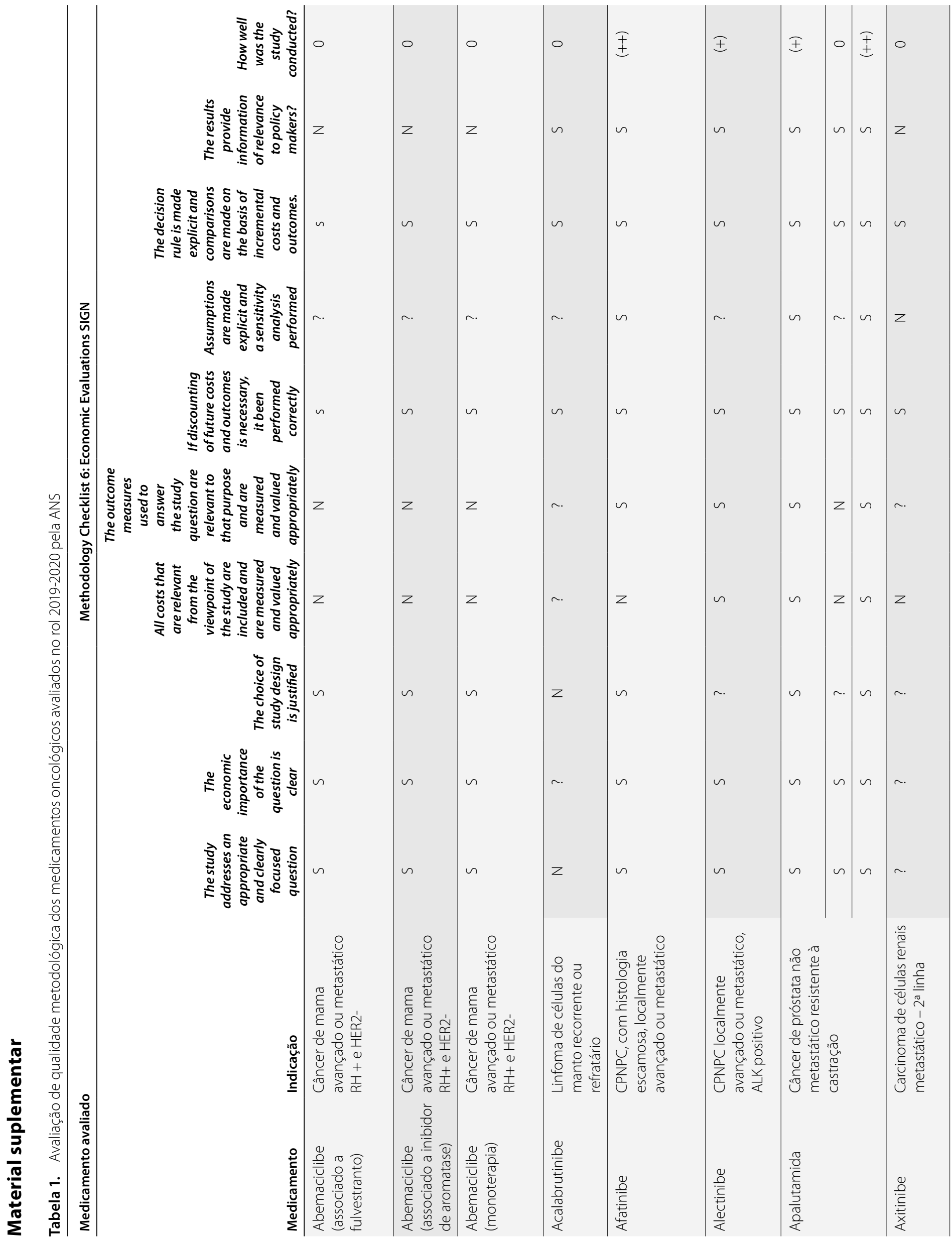




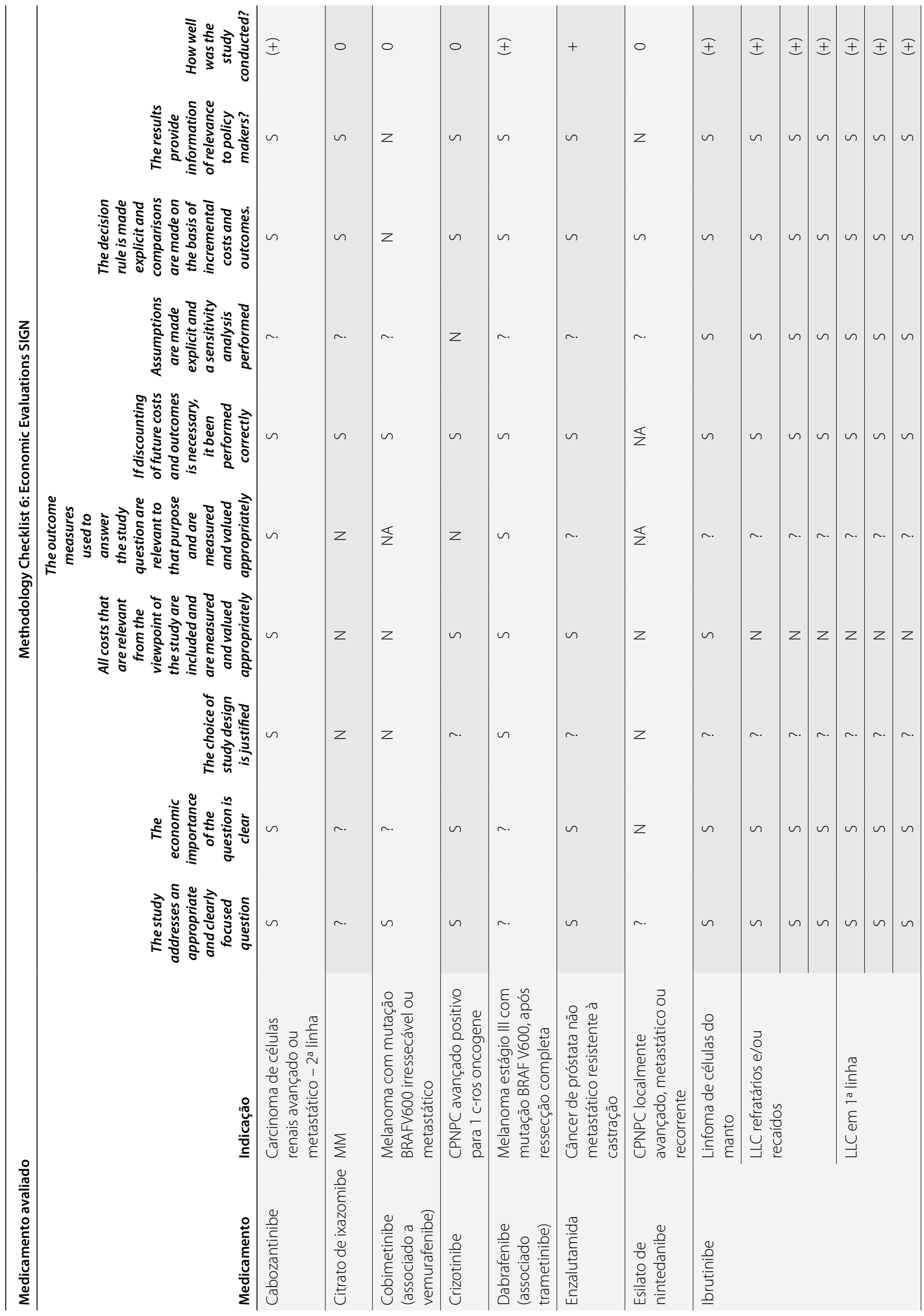




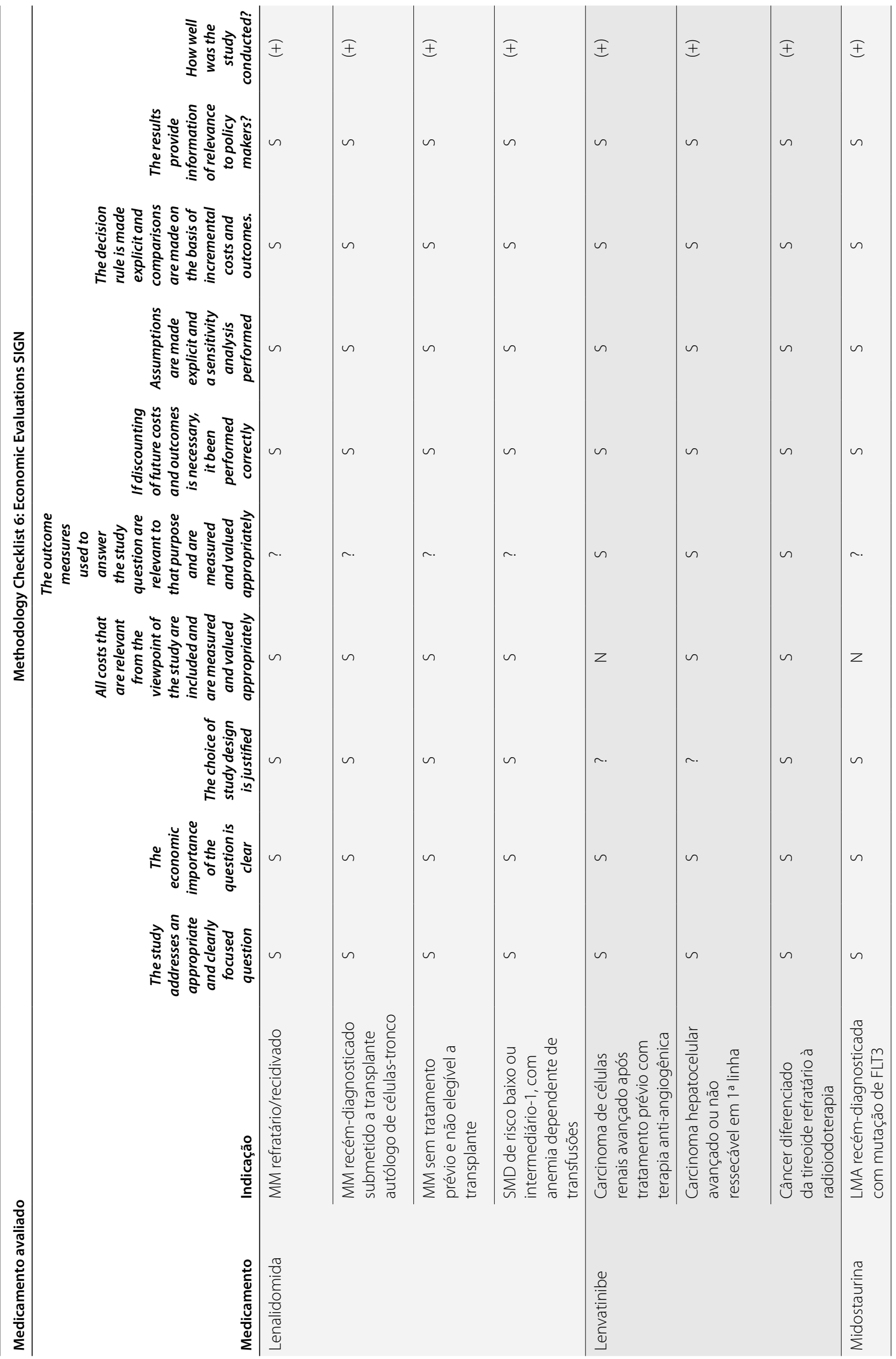




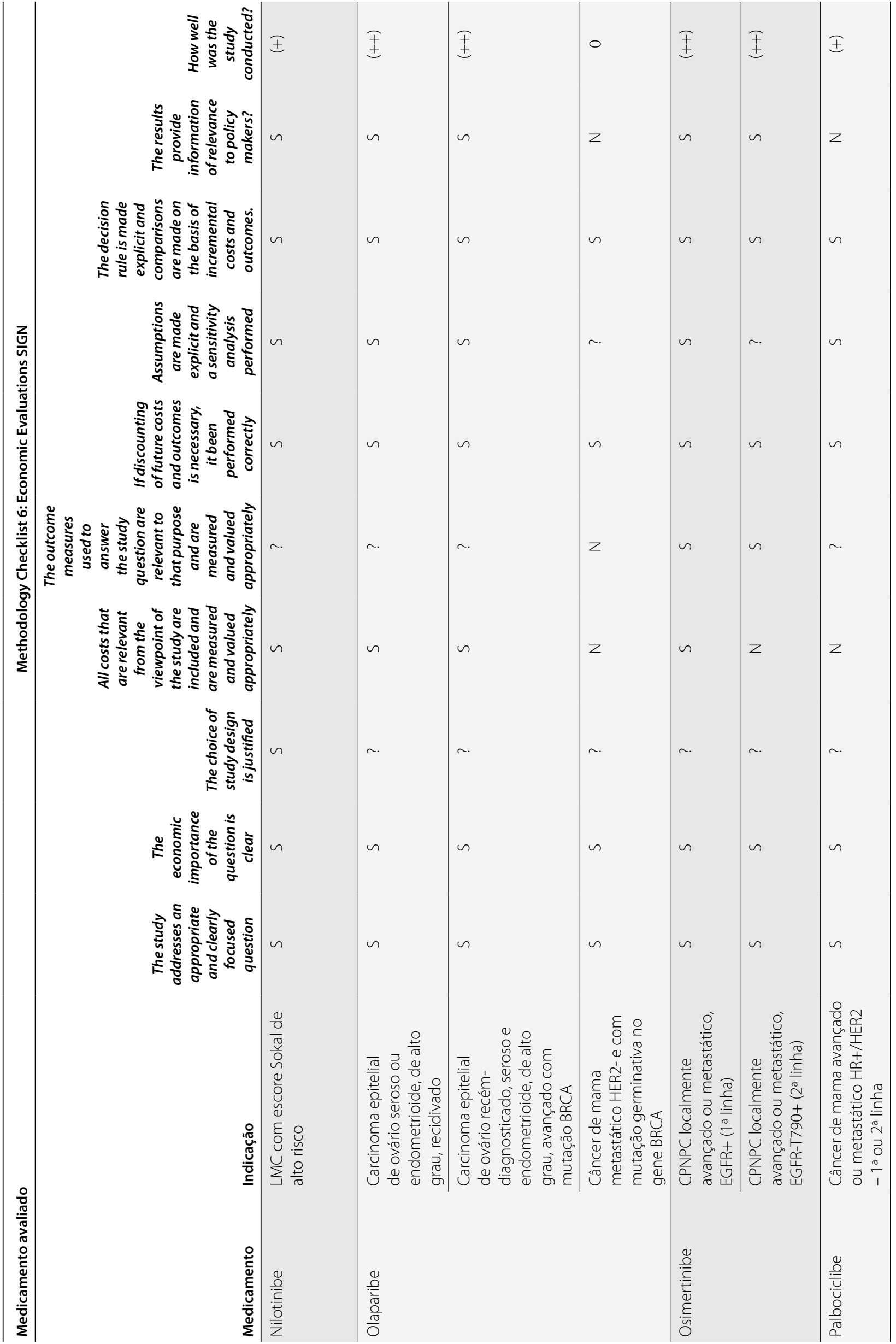




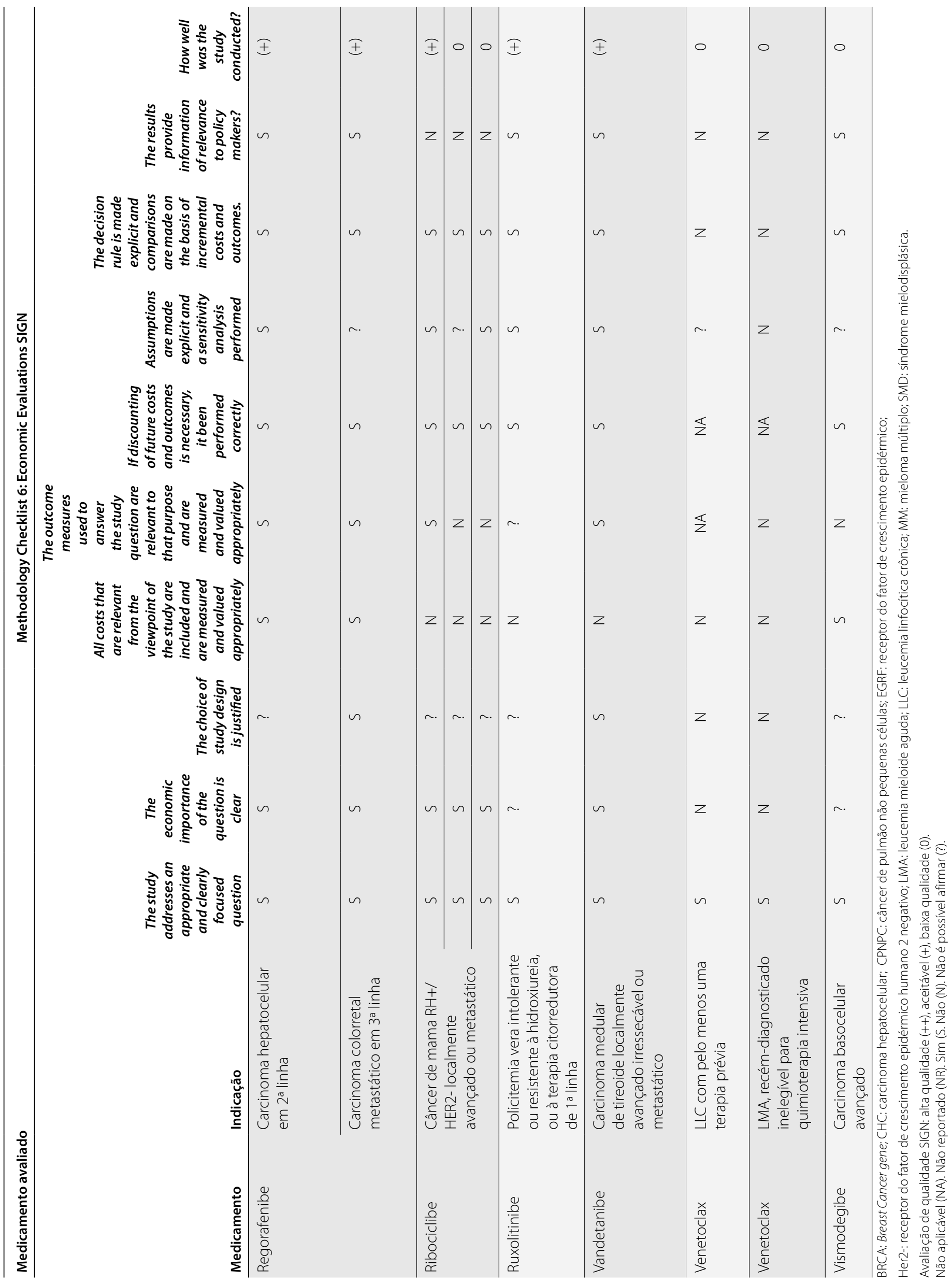

